\title{
Nanoelectromechanical infrared detector
}

\section{Markus Piller, Niklas Luhmann, Miao-Hsuan Chien, Silvan Schmid}

Markus Piller, Niklas Luhmann, Miao-Hsuan Chien, Silvan Schmid, "Nanoelectromechanical infrared detector," Proc. SPIE 11088, Optical Sensing, Imaging, and Photon Counting: From X-Rays to THz 2019, 1108802 (9 September 2019); doi: 10.1117/12.2528416

EDent: SPIE Nanoscience + Engineering, 2019, San Diego, California, United States 


\title{
Nanoelectromechanical Infrared Detector
}

\author{
Markus Piller, Niklas Luhmann, Miao-Hsuan Chien, and Silvan Schmid \\ Institute of Sensor and Actuator Systems, TU Wien, 1040 Vienna, Austria
}

\begin{abstract}
The sensitive detection of infrared (IR) radiation is a essential task in today's modern world. The sensitivity of the state-of-the-art uncooled thermal infrared detectors is still several orders of magnitude above the fundamental photon noise limit. Thermal detectors based on temperature sensitive micro- and nanomechanical resonators are a promising approach to obtain improved thermal IR detectors. Here, we present an uncooled infrared detector based on a $1 \mathrm{~mm} \times 1 \mathrm{~mm}$ large nanoelectromechanical drum resonator made of $50 \mathrm{~nm}$ thick low-stress silicon nitride (SiN). The detector features a thin film absorber with an absorptivity of $\sim 30 \%$ over the entire mid-IR range. The detector drum is driven at its resonance frequency by means of a phase-locked loop. Absorbed IR radiation results in an observable detuning of the drum's oscillation frequency. We measured an Allan deviation of $\sigma_{A}=5.5 \times 10^{-7}$ at room temperature at a noise bandwidth of $25 \mathrm{~Hz}$. With a responsivity of $R=343 \mathrm{~W}^{-1}$ this results in a sensitivity defined as noise equivalent power (NEP) of NEP $=320 \mathrm{pW} / \mathrm{rtHz}$ for an IR beam at a wavelength of $9.5 \mu \mathrm{m}$. For this measurement, the IR beam focus spot diameter was equal to the drum size. The drum's responsivity improves by a factor of ten for a focal spot size smaller than $\sim 100 \mu \mathrm{m}$. For smaller spots the responsivity remains constant. Based on this analysis we predict a sensitivity of $\sim 30 \mathrm{pW} / \mathrm{rtHz}$ for an IR spot size smaller than $100 \mu \mathrm{m}$. The detector can be improved further by e.g. optimizing the tensile pre-stress to a lower value or by improving the absorptivity.
\end{abstract}

Keywords: Thermal detector, Infrared detector, NEMS, nanoelectromechanical system, silicon nitride drum

\section{INTRODUCTION}

The sensitive detection of photons in the infrared regime of the electromagnetic spectrum is an essential task. Because of their low power, infrared (IR) photons can not be directly detected with a photon detector. Therefore, in the infrared spectrum, thermal detectors are almost exclusively used. As thermal detectors transduce the absorbed photons into heat, the power of absorbed infrared radiation is then measured by the increase in temperature. The three basic building blocks of thermal detectors are i) a radiation absorber, ii) a thermal isolation, and iii) a thermometer. Available thermal detector types differ in the way the photothermally induced increase in temperature is measured. Some of the common thermometer techniques are photoacoustic (Golay cell), thermoresistive (bolometer), pyroelectric, or thermoelectric (thermocouple). However, to this day, the sensitivity of state-of-the-art uncooled thermal infrared detectors is still several orders of magnitude above the fundamental photon noise limit. ${ }^{1}$ The detector performance can be significantly improved by cryogenic cooling, as e.g. for liquid helium cooled bolometers. However, for the majority of applications, cryogenic cooling is impedimental and therefore uncooled thermal detectors are most widely used. The Golay cell is the most sensitive commercial uncooled thermal detector with a sensitivity of around $100 \mathrm{pW} / \mathrm{rtHz}$. Due to their lower price, pyroelectric detectors are widely used and reach typical sensitivities of the order of $1 \mathrm{nW} / \mathrm{rtHz}$.

As a promising alternative, it has been proposed to build uncooled thermal detectors based on micromechanical resonators whose resonance frequency is highly temperature sensitive. ${ }^{2}$ It has since been shown that thermal infrared detectors with a sensitivity of a few $\mathrm{nW} / \mathrm{rtHz}$ can be obtained with quartz bulk acoustic wave resonators. ${ }^{3,4}$ Similar sensitivities have been reached with piezoresistive nanoplate resonators. ${ }^{5-8}$ And sensitivities below a $\mathrm{nW} / \mathrm{rtHz}$ have been obtained with micromechanical piezoelectric bridge resonators. ${ }^{9,} 10$ For the application as IR detector arrays, nanomechanical paddle resonators offer the required small footprint. With such

Further author information: (Send correspondence to S.S.)

S.S.: E-mail: silvan.schmid@tuwien.ac.at

Optical Sensing, Imaging, and Photon Counting: From X-Rays to THz 2019, edited by Oleg Mitrofanov, Proc. of SPIE Vol. 11088, 1108802 -C The Authors. Published under a Creative Commons Attribution CC-BY 3.0 License · doi: 10.1117/12.2528416

Proc. of SPIE Vol. 11088 1108802-1 
torsional paddle resonators sensitivities of a few tens of $\mathrm{pW} / \mathrm{rtHz}$ have been obtained. ${ }^{11,12}$ Another promising design is based on micromechanical shape memory polymer resonators. ${ }^{13}$

The thermal response of all the above listed micro- and nanomechanical thermal infrared detectors is mainly coming from the thermal response of the elastic modulus of the resonator material. In contrast, in microand nanomechanical string resonators the resonance frequency is determined by the tensile stress. A change in temperature causes a strong change in stress due to the thermal expansion of the resonator, resulting in an extraordinary temperature responsivity. ${ }^{14-16}$ Nanomechanical string and drum resonators made of silicon nitride have therefore successfully been used to analyse picograms of sample material by photothermal infrared absorption spectroscopy. ${ }^{17-20}$ It has further been shown that it is possible to detect and analyse individual nanoparticles with such nanomechanical string resonators. ${ }^{21,22}$ Recently, it has been shown that it is possible to reach single-molecule sensitivity with stress-optimized silicon nitride drums, reaching a record sensitivity of a few tens of $\mathrm{fW} / \mathrm{rtHz}$ in the visible regime. ${ }^{23}$ The prospect is promising, that a nanomechanical drum resonator has an intrinsic sensitivity limit below the photon-noise limit, which for an uncooled IR detector of this size is in the order of $1 \mathrm{pW} / \mathrm{rtHz}^{24}$ A very similar detector design reaching sensitivities of $7 \mathrm{pW} / \mathrm{rtHz}$ in the visible regime has very recently been presented based on graphene drum and trampoline resonators. ${ }^{25}$

The relative responsivity, which is defined as the relative resonance frequency change per unit power, of a circular drum resonator with thickness $h$ for a point heating source in the drum center is given by ${ }^{20}$

$$
\mathrm{R}=\frac{\Delta f}{f_{0}} \approx \frac{\beta}{8 \pi} \frac{1}{h} \frac{\alpha E}{\kappa \sigma}\left(\frac{2-\nu}{1-\nu}-0.64\right)
$$

with the optical absorptivity $\beta$, the thermal expansion coefficient $\alpha$, Young's modulus $E$, thermal conductivity $\kappa$, tensile stress $\sigma$, and Poisson's ration $\nu$. This analytical model has been validated by FEM, where it was also shown that it's a good approximation for rectangular drums with a model error estimated to be below one percent. ${ }^{20}$ From (1) we can draw three main conclusions: the relative responsivity of a square drum i) improves for thin drums, ii) improves for low tensile pre-stress, and iii) is independent of its lateral size. The latter fact is of particular interest because it allows the tuning of the thermal response time of the detector, which scales with size, without affecting the responsivity. In order to obtain a high responsivity, our drum resonators are fabricated from $50 \mathrm{~nm}$ thick low-stress silicon nitride ( $\mathrm{SiN})$. The SiN drum is then covered with an absorber thin film. A schematic drawing of our detector is depicted in Figure 1.

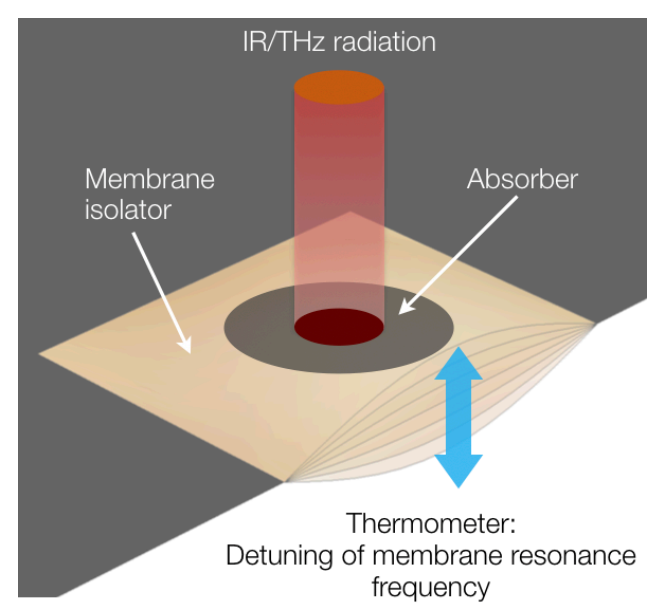

Figure 1. Schematic drawing of detector working principle. The detector is based on a $50 \mathrm{~nm}$ thick drum resonator made of silicon nitride, comprising an absorber layer. The absorber thin film thermalizes part of the radiation. The resulting photothermal heating of the nanoelectromechancial drum causes a measurable detuning of the drum's resonance frequency. 


\section{METHODS}

The infrared detector chip basically consists of a squared silicon nitride drum resonator with a fully covered absorbing layer and metal structures for electronic transduction. The fabrication is based on single silicon wafer with a thickness of $370 \mu \mathrm{m}$ and a double side coating by use of low pressure chemical vapor deposition (LPCVD) with a $50 \mathrm{~nm}$ thick layer of nonstoichiometric silicon nitride (SiN). Initially the tensile stress of the SiN layer was $150 \mathrm{MPa}$. A conventional UV lithography was used for defining the detector chip structures in a photoresist. The metal electrodes $(\mathrm{Cr} / \mathrm{Au}=5 \mathrm{~nm} / 100 \mathrm{~nm})$ on top of the drum resonator were deposited by use of a physical vapor deposition (PVD) process with chromium as adhesion layer. The SiN drum was structured from the backside, first with a reactive ion etching (RIE) step removing the backside SiN layer for the $1 \mathrm{~mm} \times 1 \mathrm{~mm}$ drum window opening. In a second step the underlying silicon was etched with potassium hydroxide (KOH). An absorbing thin film of $\sim 14 \mathrm{~nm}$ was deposited to the final $\mathrm{SiN}$ drum resonator from the backside.

A microscope image of the SiN drum with which the presented results were obtained is shown in Figure 2a. The inductive transduction was integrated with two separate Au electrodes, one for actuation and one for detection. Figure $2 \mathrm{~b}$ represents the absorption spectrum of the SiN/absorber drum, measured by FTIR. The detector has an absorptivity of $\beta \sim 0.32$ over a broad spectral range from $5 \mu \mathrm{m}$ to $20 \mu \mathrm{m}$.
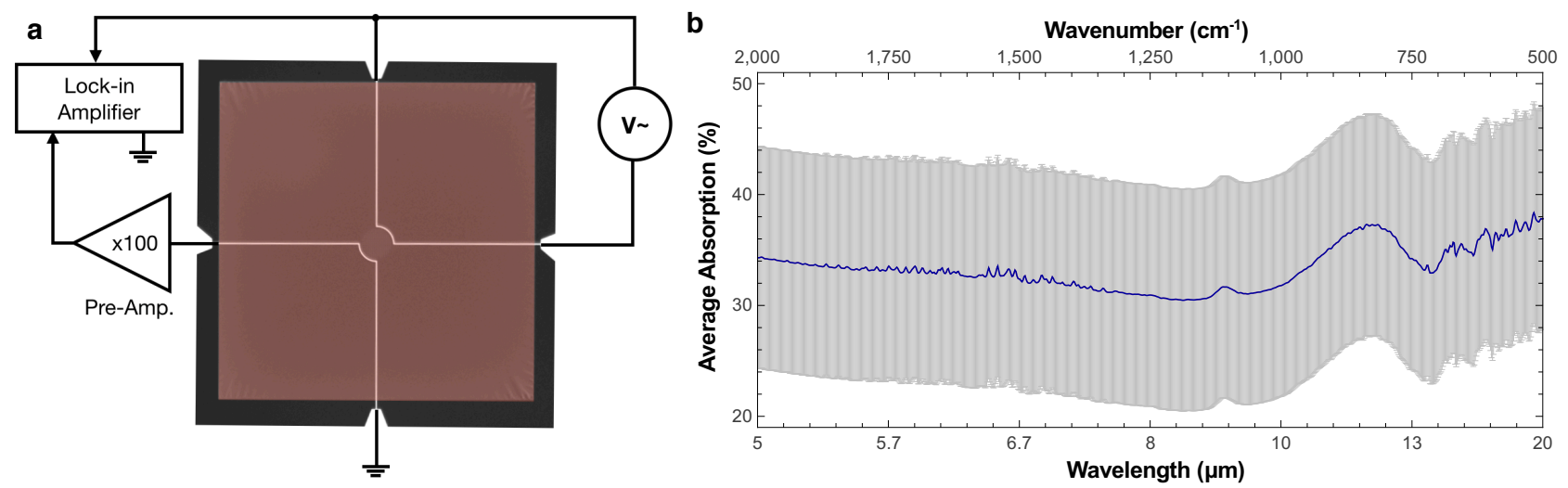

Figure 2. a) Microscope image of a $1 \times 1 \mathrm{~mm}$ large and $50 \mathrm{~nm}$ thick SiN drum. The drum features Au electrodes used for the inductive transduction. The entire backside of the SiN drum is coated with a thin film as IR absorber. Schematics of the electronic connections are added to the image. b) Absorption spectrum of our detector, derived from combined FTIR transmission and reflection measurements.

A schematic of the experimental setup is shown in Figure 3. The measurements were performed in vacuum at a pressure below $1 \times 10^{-3} \mathrm{mbar}$. The drum resonator was operated in an oscillator mode produced by a phase-locked loop (PLL), created with a lock-in amplifier (Zurich Instrumentsm, HF2LI). The infrared light was produced by a globar light source (Arcoptix ArcLight IR). All measurements were performed with a bandpass filter $(9.5 \mu \mathrm{m}$ and bandwidth of $500 \mathrm{~nm})$ in the IR light path.

\section{RESULTS \& DISCUSSION}

Figure $4 \mathrm{a}$ shows the frequency signal over time. From the corresponding Allan deviation curve in Figure $4 \mathrm{~b}$ an optimal value of $\sigma_{A}=5.5 \times 10^{-7}$ at a noise bandwidth of $25 \mathrm{~Hz}$ can be extracted. The frequency response of the drum to IR light with a wavelength of $9.5 \mu \mathrm{m}$ is shown in figure 5a). The incident power of $800 \mathrm{nW}$ causes a frequency detuning of $\sim 20 \mathrm{~Hz}$. This results in a measured relative responsivity of $R=20 \mathrm{~Hz} / 72940 \mathrm{~Hz} / 800 \mathrm{nW}=343 \mathrm{~W}^{-1}$. follows

One figure of merit for an optical detector is the noise equivalent power (NEP), which can be calculated as

$$
\mathrm{NEP}=\frac{\sigma_{A} \sqrt{\tau}}{R} .
$$

Based on (2) one can now calculate the resulting NEP $=5.5 \times 10^{-7} \sqrt{0.04 \mathrm{~s}} / 343 \mathrm{~W}^{-1}=320 \mathrm{pW} / \mathrm{rtHz}$. This NEP corresponds to the situation where the IR spot is fully filling the drum area. From figure $6 \mathrm{~b}$ we can see, 

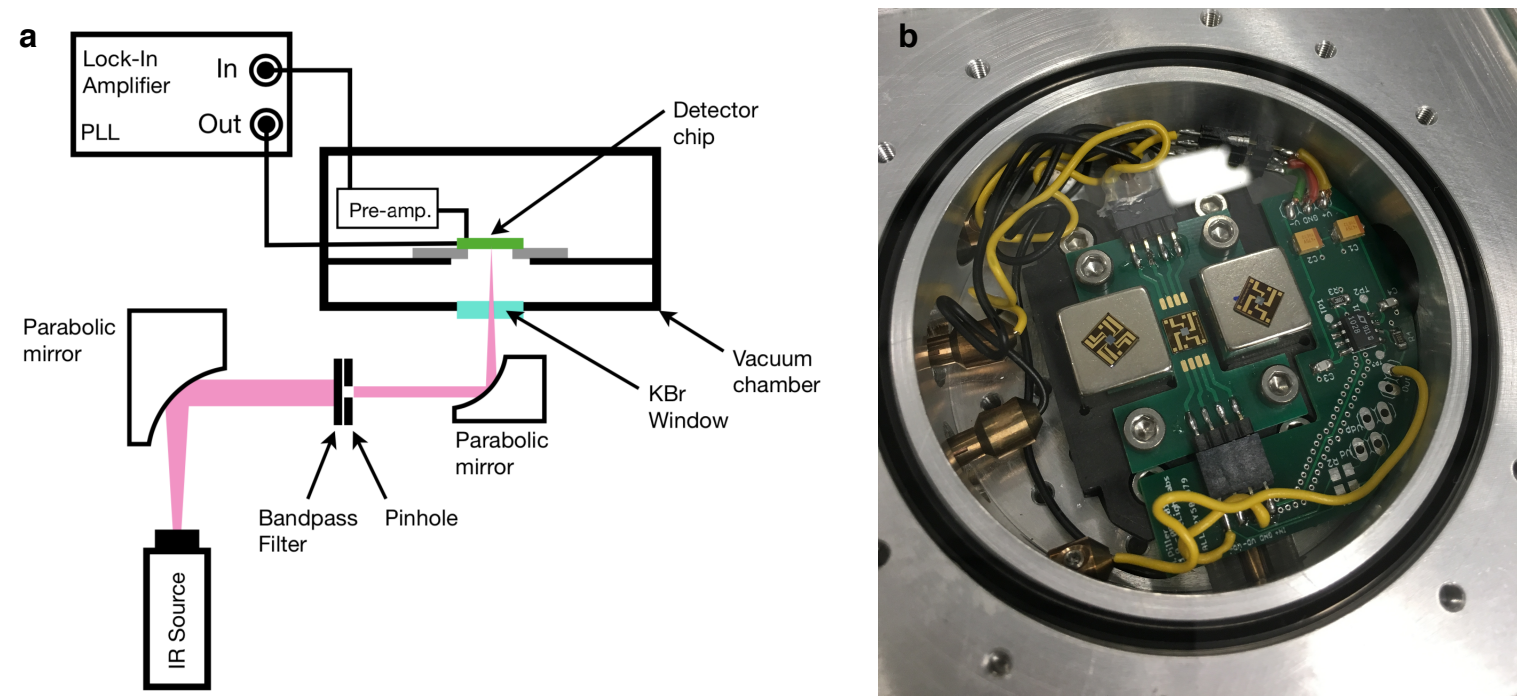

Figure 3. a) Schematic drawing of the experimental setup. b) Photograph of the detector chip sitting in-between two magnets inside a vacuum chamber.
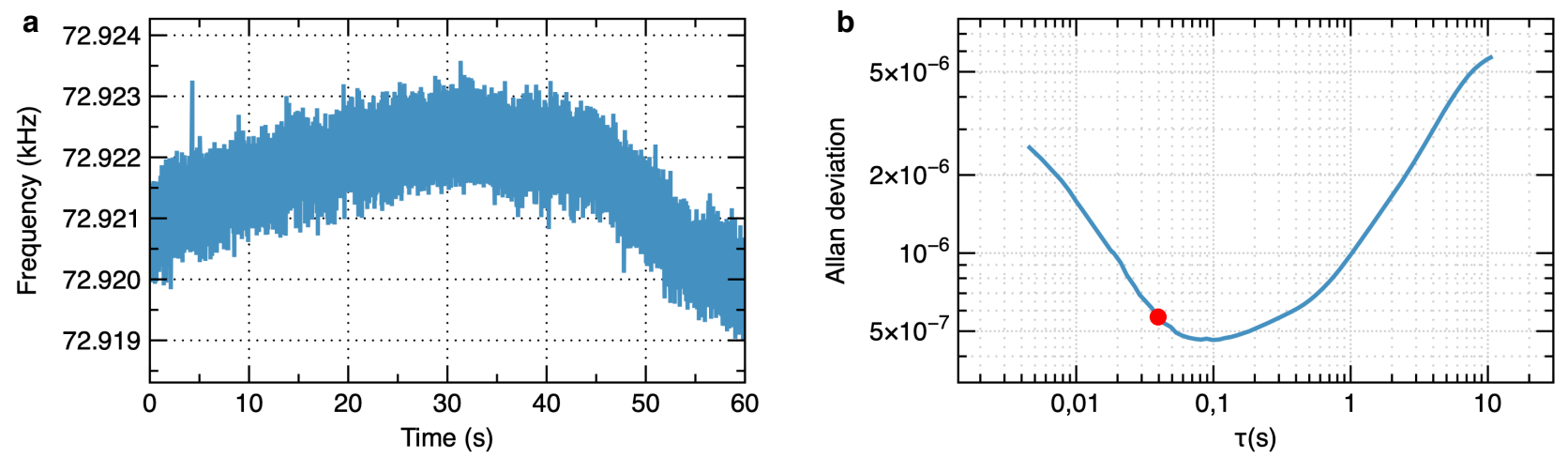

Figure 4. a) Frequency signal of $1 \times 1 \mathrm{~mm}$ large and $50 \mathrm{~nm}$ thick SiN drum over time (sample shown in Figure 2a). b) Respective Allan deviation $\sigma_{f}$.

that the beam spot size has a large effect on the responsivity. In fact, assuming an IR beam spot size smaller than $\sim 100 \mu \mathrm{m}$, the responsivity would be roughly a factor of ten higher. Hence, for an optimized IR spot size an $\mathrm{NEP} \approx 30 \mathrm{pW} / \mathrm{rtHz}$ could be expected.

The thermal response time of the SiN drum detector was analysed by the "90/10 method". ${ }^{26}$ Therefore the rise time was extracted from a step signal taking the temporal difference were 10 and 90 percent of the frequency shift are reached, as shown in figure 5b). The fall time of $\tau_{r}=35 \mathrm{~ms}$ results in a response time corresponding to a first order low-pass filter of $\tau=\tau_{r} / \ln (9)=17 \mathrm{~ms}$. The obtained time constant of $\tau=17 \mathrm{~ms}$ is faster than the $40 \mathrm{~ms}$ integration time required to reach the optimum Allan deviation. Hence, the presented detector is frequency limited by the noise bandwidth.

\section{CONCLUSION}

We have demonstrated the application of SiN drum resonators as uncooled nanoelectromechanical IR detector. The detector principle is based on the thermally induced detuning of the resonance frequency. Comprising a broad spectral absorber, we obtained a NEP of $320 \mathrm{pW} / \mathrm{rtHz}$ over the IR range from $5 \mu \mathrm{m}$ to $20 \mu \mathrm{m}$. FEM simulations of the IR spot size and related thermomechanic response show, that the NEP can be significantly 
a

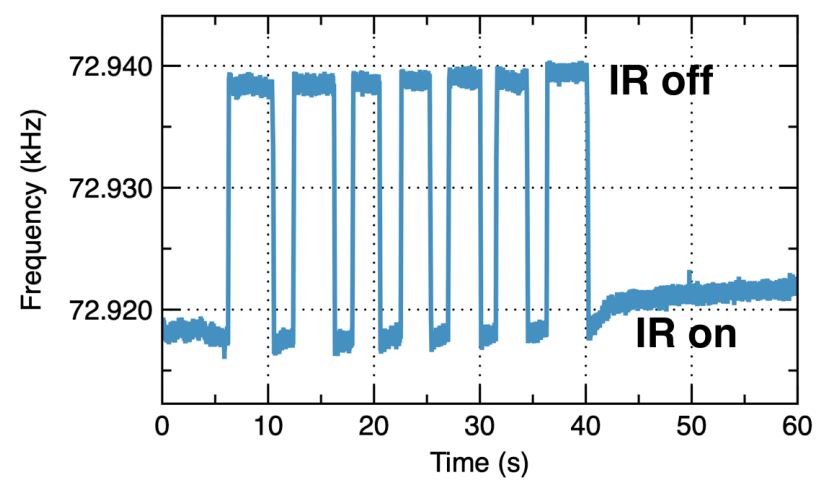

b

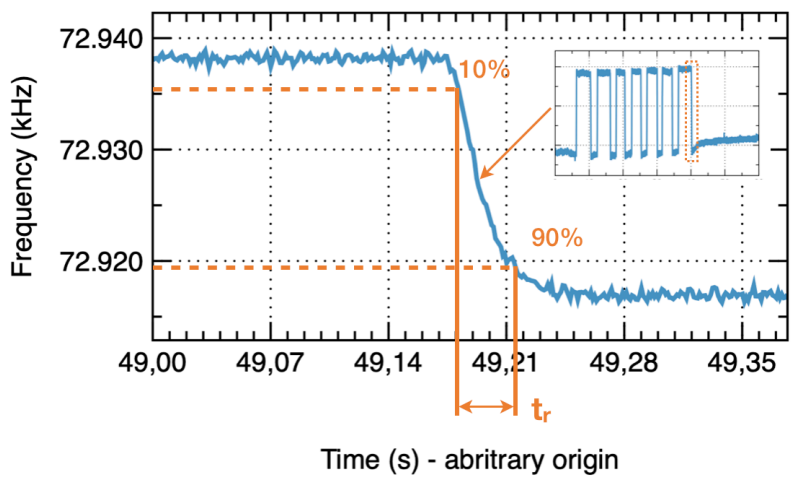

Figure 5. a) Frequency response of a $1 \times 1 \mathrm{~mm}$ large and $50 \mathrm{~nm}$ thick SiN drum (sample shown in Figure $2 \mathrm{a}$ ) to an IR beam of $800 \mathrm{nW}$ incident power with a wavelength of $9.5 \mu \mathrm{m}$ and spot size of $1 \mathrm{~mm}$. b) "90/10 method" for extracting the rise time from the measured frequency response to a change in incident power.
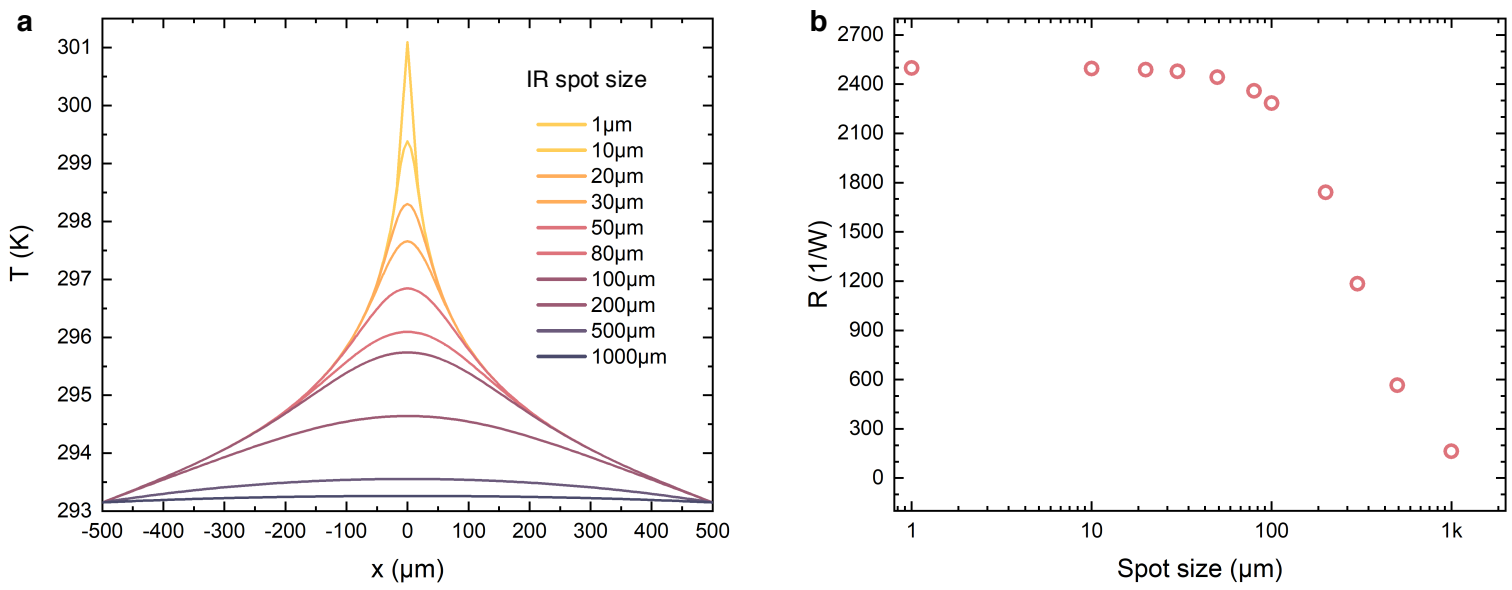

Figure 6. a) FEM simulation of the temperature profile across a $1 \mathrm{~mm}$ large and $50 \mathrm{~nm}$ thick SiN drum for an IR beam with fixed power but varying spot size (assuming a Gaussian beam profile). b) Corresponding relative responsivity as a function of the IR spot size.

improved by reducing the incident beam diameter. Considering a reduction of the spot size to less than $100 \mu \mathrm{m}$, an NEP of $30 \mathrm{pW} / \mathrm{rtHz}$ could be expected. The detector responsivity can be further improved by lowering the initial tensile stress of the SiN using for instance oxygen plasma or sputtered thin films with compressive stress. $^{27,28}$ Assuming the stress reduction process does not change the noise values, an enhanced responsivity can further improve the sensitivity towards the fundamental limit. ${ }^{2}$

\section{ACKNOWLEDGMENTS}

We thank Michael Feiginov and Josiane P. Lafleur for the helpful discussions, and Johannes Steurer for his support with the electronic design. We further thank Sophia Ewert, Michael Buchholz, and Patrick Meyer for their assistance with device microfabrication. This work has received funding from the company Invisible-Light Labs GmbH. This work has received funding from the European Research Council under the European Unions Horizon 2020 research and innovation program (Grant Agreement-716087-PLASMECS). 


\section{REFERENCES}

[1] Rogalski, A., [Infrared Detectors], CRC Press, 2nd ed. (2011).

[2] Vig, J. R., Filler, R. L., and Kim, Y., "Uncooled IR imaging array based on quartz microresonators," Journal of Microelectromechanical Systems 5(2), 131-137 (1996).

[3] Pisani, M. B., Ren, K., Kao, P., and Tadigadapa, S., "Application of Micromachined y -cut-quartz bulk acoustic wave resonator for infrared sensing," Journal of Microelectromechanical Systems 20(1), 288-296 (2011).

[4] Kao, P. and Tadigadapa, S., "Micromachined quartz resonator based infrared detector array," Sensors and Actuators, A: Physical 149(2), 189-192 (2009).

[5] Gokhale, V. J. and Rais-Zadeh, M., "Uncooled infrared detectors using gallium nitride on silicon micromechanical resonators," Journal of Microelectromechanical Systems 23(4), 803-810 (2014).

[6] Qian, Z., Hui, Y., Liu, F., Kang, S., Kar, S., and Rinaldi, M., "GrapheneAluminum nitride NEMS resonant infrared detector," Microsystems and Nanoengineering 2(January) (2016).

[7] Hui, Y., Gomez-Diaz, J. S., Qian, Z., Alù, A., and Rinaldi, M., "Plasmonic piezoelectric nanomechanical resonator for spectrally selective infrared sensing.," Nature communications 7, 11249 (2016).

[8] Hui, Y. and Rinaldi, M., "High performance NEMS resonant infrared detector based on an aluminum nitride nano-plate resonator," 2013 Transducers and Eurosensors XXVII: The 17th International Conference on Solid-State Sensors, Actuators and Microsystems, TRANSDUCERS and EUROSENSORS 2013 (June), 968-971 (2013).

[9] Zhang, Y., Hosono, S., Nagai, N., Song, S. H., and Hirakawa, K., "Fast and sensitive bolometric terahertz detection at room temperature through thermomechanical transduction," Journal of Applied Physics 125(15) (2019).

[10] Zhang, Y., Watanabe, Y., Hosono, S., Nagai, N., and Hirakawa, K., "Room temperature, very sensitive thermometer using a doubly clamped microelectromechanical beam resonator for bolometer applications," Applied Physics Letters 108(16) (2016).

[11] Zhang, X. C., Myers, E. B., Sader, J. E., and Roukes, M. L., "Nanomechanical torsional resonators for frequency-shift infrared thermal sensing.," Nano letters 13, 1528-34 (apr 2013).

[12] Laurent, L., Yon, J. J., Moulet, J. S., Roukes, M., and Duraffourg, L., "12- $\mu$ m -Pitch Electromechanical Resonator for Thermal Sensing," Physical Review Applied 9(2), 1-11 (2018).

[13] Adiyan, U., Larsen, T., Zárate, J. J., and Villanueva, L. G., "Shape Memory Polymer Resonators as Highly Sensitive Uncooled Infrared Detectors," arXiv:1904.01453 [physics.ins-det] (2019).

[14] Pandey, A. K., Gottlieb, O., Shtempluck, O., and Buks, E., "Performance of an AuPd micromechanical resonator as a temperature sensor," Applied Physics Letters 96, 203105 (2010).

[15] Larsen, T., Schmid, S., Gronberg, L., Niskanen, A. O., Hassel, J., Dohn, S., and Boisen, A., "Ultrasensitive string-based temperature sensors," Applied Physics Letters 98, 121901 (2011).

[16] Larsen, T., Schmid, S., and Boisen, A., "Micro string resonators as temperature sensors," in [AIP Conference Proceedings], 1552 8, 931-936 (2013).

[17] Yamada, S., Schmid, S., Larsen, T., Hansen, O., and Boisen, A., "Photothermal infrared spectroscopy of airborne samples with mechanical string resonators," Analytical Chemistry 85, 10531-10535 (2013).

[18] Andersen, A. J., Yamada, S., Kumar E.K., P., Andresen, T. L., Boisen, A., and Schmid, S., "Nanomechanical IR spectroscopy for fast analysis of liquid-dispersed engineered nanomaterials," Sensors and Actuators B: Chemical 233, 667-673 (2016).

[19] Biswas, T. S., Miriyala, N., Doolin, C., Liu, X., Thundat, T., and Davis, J. P., "Femtogram-Scale Photothermal Spectroscopy of Explosive Molecules on Nanostrings," Analytical chemistry 86, 11368-11372 (2014).

[20] Kurek, M., Carnoy, M., Larsen, P. E., Nielsen, L. H., Hansen, O., Rades, T., Schmid, S., and Boisen, A., "Nanomechanical Infrared Spectroscopy with Vibrating Filters for Pharmaceutical Analysis," Angewandte Chemie International Edition 56, 3901-3905 (2017).

[21] Larsen, T., Schmid, S., Villanueva, L. G., and Boisen, A., "Photothermal analysis of individual nanoparticulate samples using micromechanical resonators.," ACS Nano 7, 6188-6193 (jul 2013). 
[22] Schmid, S., Wu, K., Larsen, P. E., Rindzevicius, T., and Boisen, A., "Low-power photothermal probing of single plasmonic nanostructures with nanomechanical string resonators.," Nano Letters 14, 2318-2321 (apr 2014).

[23] Chien, M.-H., Brameshuber, M., Rossboth, B. K., Schütz, G. J., and Schmid, S., "Single-molecule optical absorption imaging by nanomechanical photothermal sensing," Proceedings of the National Academy of Sciences 115(44), 11150-11155 (2018).

[24] Kruse, P. W., [Uncooled Thermal Imaging] (2001).

[25] Blaikie, A., Miller, D., and Alemán, B. J., "A fast, sensitive, room-temperature graphene nanomechanical bolometer," arXiv:1810.13422 [cond-mat.mes-hall] (2018).

[26] Duraffourg, L., Laurent, L., Moulet, J. S., Arcamone, J., and Yon, J. J., "Array of resonant electromechanical nanosystems: A technological breakthrough for uncooled infrared imaging," Micromachines 9(8) (2018).

[27] Luhmann, N., Jachimowicz, A., Schalko, J., Sadeghi, P., Sauer, M., Foelske-schmitz, A., and Schmid, S., "Effect of oxygen plasma on nanomechanical silicon nitride resonators," Applied Physics Letters 111, 063103 (2017).

[28] Windischmann, H., "Intrinsic stress in sputter-deposited thin films," Critical Reviews in Solid State and Materials Sciences 17(6), 547-596 (1992). 